\title{
THE EFFECT OF RETINOIC ACID ON HUMAN ADRENAL CORTICOSTEROID SECRETION IN VITRO
}

Antonella Sesta ${ }^{1}$, Laura Tapella ${ }^{2}$, Maria Francesca Cassarino ${ }^{1}$, Luigi Castelli ${ }^{3}$, Francesco Cavagnini ${ }^{1}$, Francesca Pecori Giraldi ${ }^{1,2}$,

${ }^{1}$ Neuroendocrinology Research Lab, Istituto Auxologico Italiano IRCCS, Milan;

${ }^{2}$ Dept. Clinical Sciences \& Community Health, University of Milan; ${ }^{3}$ Clinica San Carlo, Paderno Dugnano, Italy

\section{INTRODUCTION}

Retinoic acid, a derivative of vitamin A, has recently yielded promising results in the treatment of Cushing's disease (Pecori Giraldi et al JCEM 2012). Its main site of action appears to be the tumoral corticotrope as retinoic acid inhibits POMC transcription and corticotrope proliferation (Paez-Pereda et al JCI 2001). Studies on tumoral adrenal cell lines have revealed an additional inhibitory effect on cell proliferation and stimulated corticosteroid secretion (Paez-Pereda et al JCI 2001).

$\underline{\text { Aim }}$ of the current study was to evaluate whether retinoic acid modulates corticosteroid secretion and genes involved in steroidogenesis pathway by normal adrenals in vitro.

\section{METHODS}

Primary cultures from 9 normal human adrenals were incubated with $10 \mathrm{nM}, 100 \mathrm{nM}$ and $1 \mu \mathrm{M}$ retinoic acid with and without $10 \mathrm{nM} \mathrm{ACTH} \mathrm{for} 24$ hours. Control wells were incubated with plain medium. Cortisol levels in medium were measured by Coat-A-Count RIA (Siemens Healthcare Diagnostics, Erlangen, Germany); CYP11A, STAR and MC2R expression were analyzed by real-time PCR (7900 HT Sequence Detection System, Applied Biosystems, Foster City, USA) normalized to RPLPO.

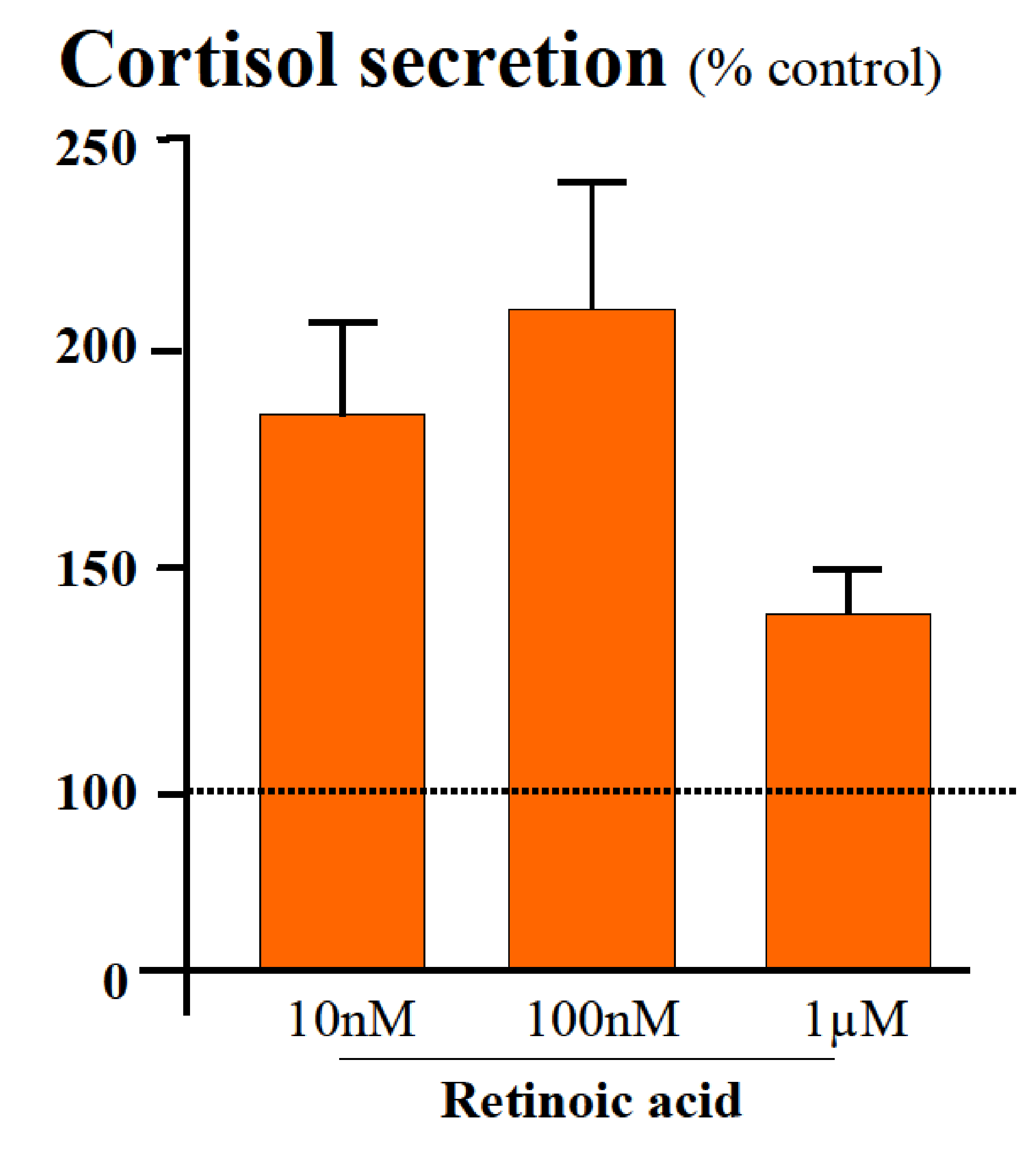

A clear-cut increase in spontaneous cortisol secretion was observed in $\mathbf{5}$ adrenal specimens.

\section{RESULTS}

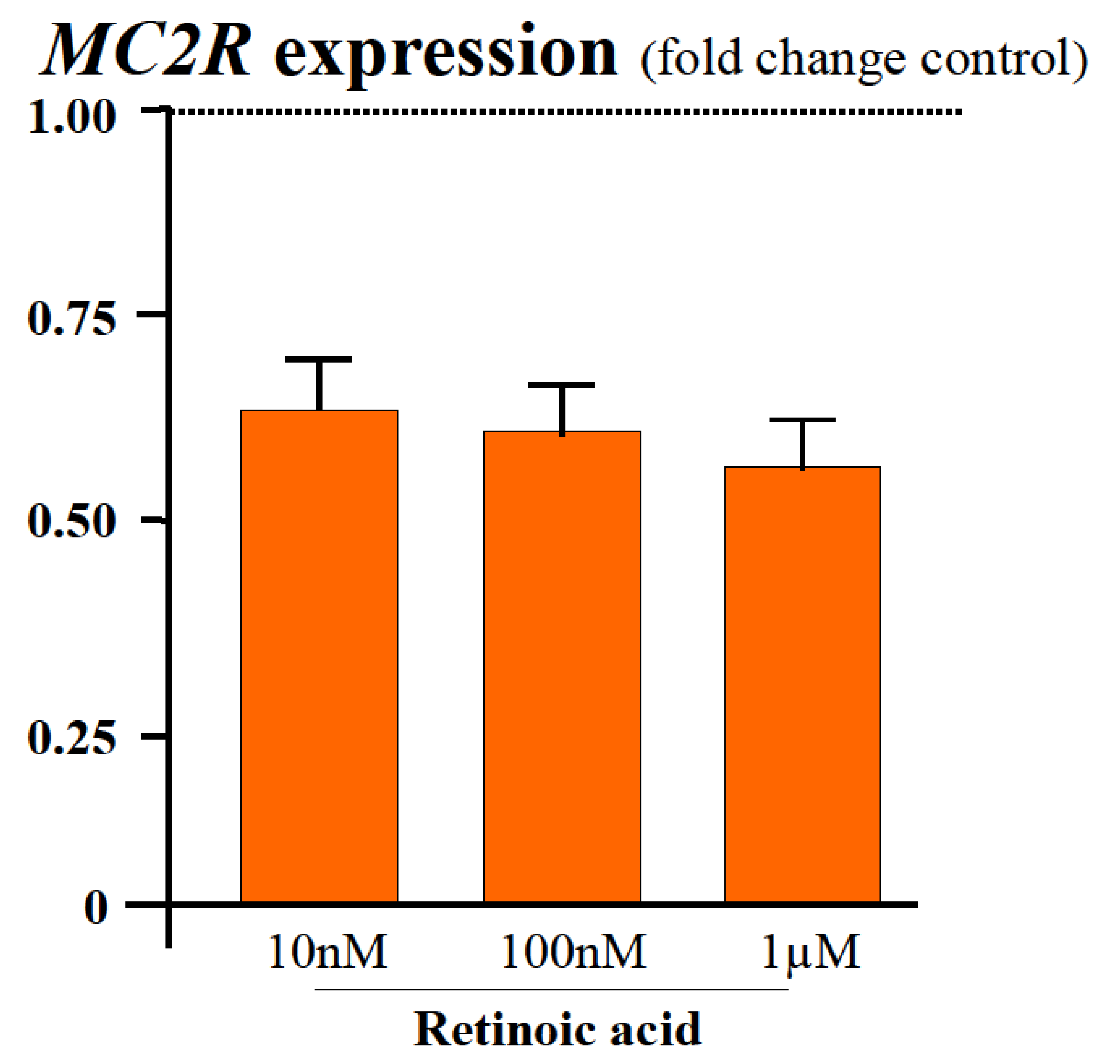

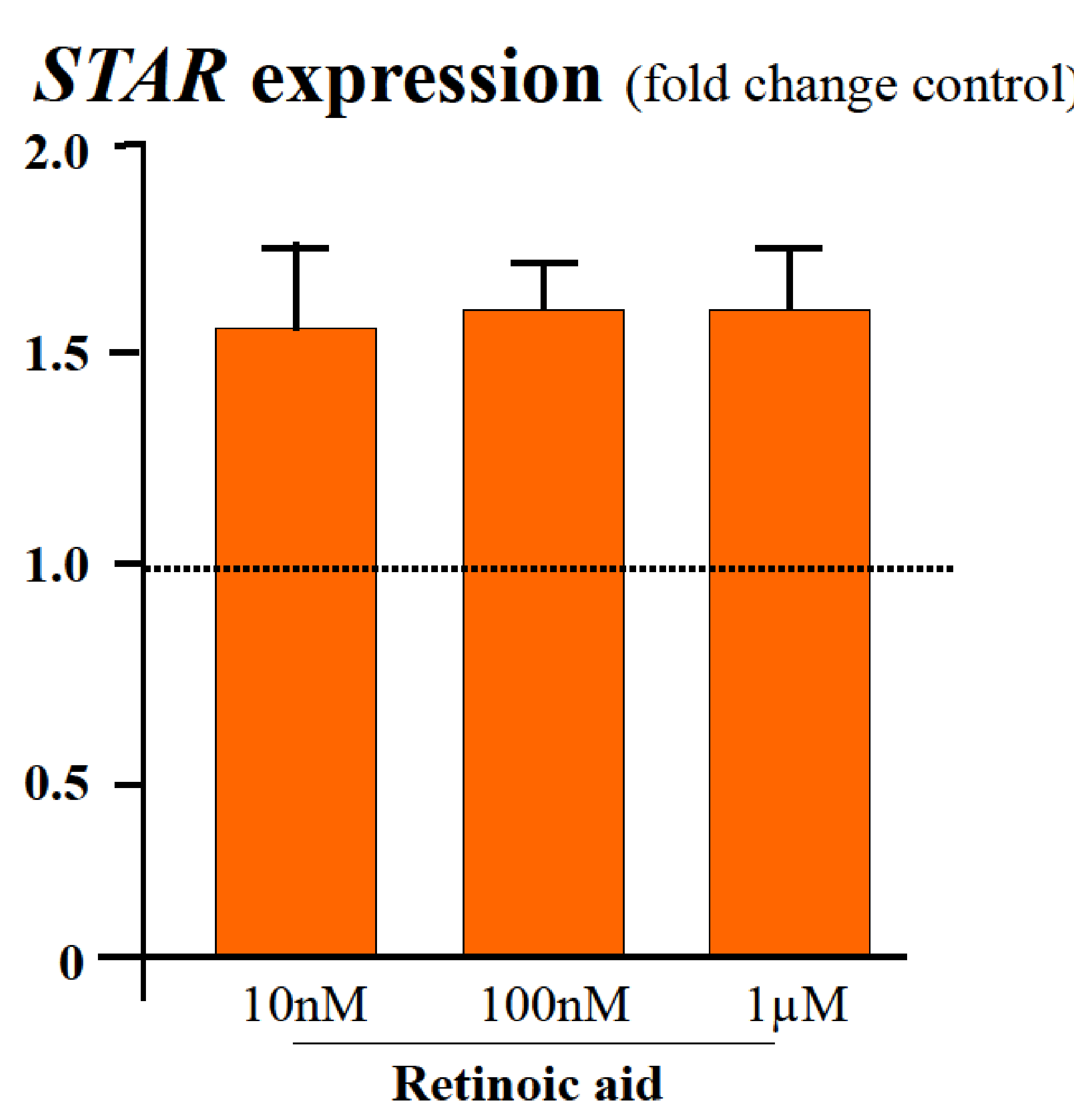

Gene expression analysis revealed a marked decrease in $M C 2 R$ expression and an increase in STAR in wells treated with retinoic acid. CYP11A expression was unchanged by retinoic acid.
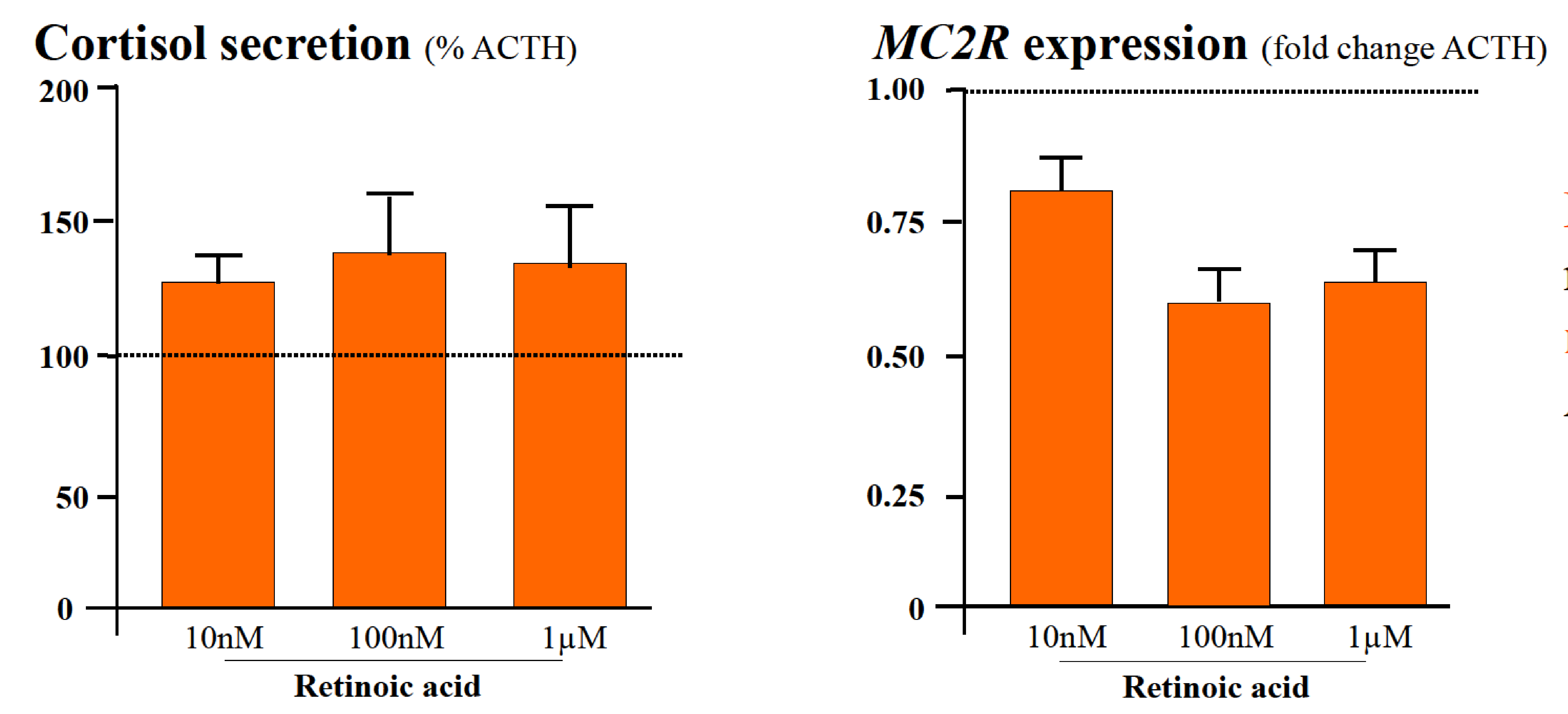

Retinoic acid and ACTH co-incubation resulted in a sligthly greater cortisol release and $M C 2 R$ inhibition than ACTH alone

\section{CONCLUSIONS}

Retinoic acid exerts a stimulatory effect on adrenal corticosteroid secretion in vitro, activates STAR expression and blunts $M C 2 R$ transcription. These findings pave the way to novel avenues of research in patients with Cushing's syndrome. 